This PDF is a selection from an out-of-print volume from the National Bureau of Economic Research

Volume Title: Inflation, Tax Rules, and Capital Formation

Volume Author/Editor: Martin Feldstein

Volume Publisher: University of Chicago Press

Volume ISBN: 0-226-24085-1

Volume URL: http://www.nber.org/books/feld83-1

Publication Date: 1983

Chapter Title: Inflation, Portfolio Choice, and Prices of Land and Corporate Stock

Chapter Author: Martin Feldstein

Chapter URL: http://www.nber.org/chapters/c11338

Chapter pages in book: (p. 229 - 242) 


\section{Inflation, Portfolio Choice, and the Prices of Land and Corporate Stock}

During the rapid inflation of the past decade, the price of land has not only kept its real value but has increased far more rapidly than the general price level. ${ }^{1}$ While elementary economic theory would predict that land and all other real assets would hold their real value when the price level rose, the increase in the relative price of land caught economists as well as others by surprise.

The reasons for the rise in the relative price of land are multiple and complex. They range from the rise in the world price of food to the political instability in the Middle East and the fears of political change in Western Europe. No single paper, let alone a short theoretical one, could hope to provide a full explanation.

There is, however, a fundamental link between general price inflation and the relative price of land that deserves particular attention. This relation is the opposite side of the same coin that causes inflation to depress the price of common stock. In essence, inflation and the tax laws interact to raise the return on land and lower the return on reproducible capital. ${ }^{2}$ The prices of these assets must then adjust to the new inflation expectation to make investors willing to hold both types of assets in the initially existing quantities. This requires the price of land to rise (relative to the general price level) and the price of reproducible capital to fall.

Reprinted by permission from American Journal of Agricultural Economics 62 (December 1980): 910-16.

1. For the 1970 s as a whole, the Agriculture Department's index of the price of farm land rose at an annual rate of 13 percent, nearly double the 7.4 percent annual rise in the general consumer price index.

2. In this paper, I use the term reproducible capital to refer to business capital and ignore owner-occupied housing. In many ways, owner-occupied housing behaves like land in its response to inflation. 
If uncertainty could be ignored, the price changes would be such that the real after-tax rates of return were equal both before and after any change in the rate of inflation. A model of asset demand that makes this simple arbitrage assumption and ignores uncertainty can, however, be very misleading. The present paper presents an explicit model of portfolio demand and uses it to show how the rate of inflation and its variance affect the real prices of land and capital.

The present paper is thus an extension of two earlier studies in which I presented models of how the interaction of inflation and the tax rules alters the real prices of land (Feldstein, 1980; chap. 12 above) and common stock (Feldstein, 1980b, $d$; chaps. 10-11). Although these papers considered the role of uncertainty in a rather ad hoc way, a formal model of portfolio choice derived from utility maximization was lacking. The purpose of the present paper is to remedy that deficiency.

A basic result of the earlier papers (as well as of the present analysis) is that changes in the rate of inflation alter the relative price of assets while at any constant inflation rate the equilibrium real asset prices remain unchanged. Thus an unanticipated jump in the rate of inflation causes an immediate jump in the level of the land price. After this initial jump, the price of land increases at the same rate as the general rate of inflation.

This interpretation implies that the continuous increase in the price of land during the $1970 \mathrm{~s}$ can best be thought of as a combination of (1) many small changes in the equilibrium real price of land (as the expected rate of general price inflation changed) and (2) a continuing increase in the nominal price of land at the prevailing rate of inflation. Similarly, the fall in the real value of share prices combines a series of falls in the equilibrium real price of shares with continuous increases in their nominal price.

The first section of this paper presents the model of portfolio equilibrium while the second section derives the means and variances of the asset yields. The price equations for land and reproducible capital are then developed in section 13.3. The fourth section derives the comparative static results for changes in inflation and in the uncertainty of inflation. A brief concluding section discusses some of the implications of this work and possible directions for further research.

\subsection{A Model of Portfolio Equilibrium}

The economy that I shall describe consists of identical individuals ${ }^{3}$ who hold a short term nominal asset ("bills"), land, and (reproducible) capi-

3. The assumption of identical individuals ignores another important feature that belongs in a more complete model of portfolio choice: differences in tax rates among investors. The distinction between taxable individual investors and tax exempt institutions can be particularly important in understanding the effect of inflation on portfolio investment (Feldstein, 1980b, $d$; chaps. 10 and 11 above). 
tal. The current price level and current inflation rate are known but the rate of inflation in the future is unknown. For simplicity, it is easiest to think of the economy switching from one expected inflation rate to another. ${ }^{4}$

The aggregate stocks of both land and capital are assumed fixed. While this may be a realistic approximation for land, ${ }^{5}$ it is clearly not an appropriate model for capital. If the market price of existing capital assets ${ }^{6}$ falls below replacement cost, the size of the capital stock will fall while a market price of existing assets above their replacement cost will cause an increase in net investment. The anticipation of the future change in the size of the capital stock will change the expected future yields per unit of capital and labor. That, in turn, will influence the initial changes in the prices of these assets. While it would clearly be desirable to incorporate this effect into the analysis, the combination of dynamic price adjustments and explicit portfolio choice under uncertainty is a more complex problem than I can currently solve. ${ }^{7}$ I have chosen to focus on the portfolio choice aspect but I recognize the importance of extending the specification to incorporate the dynamic general equilibrium response.

Consider an individual $i$ whose initial holdings of land, capital and money are $\bar{L}_{i}$ units of land, $\bar{K}_{i}$ units of capital, and $\bar{B}_{i}$ dollars of Treasury bills. These holdings reflect some previous set of expectations about asset yields and the associated covariance matrix. When the Hicksian "week" begins, there is a new set of expectations (possibly but not necessarily identical with the old ones). These expectations imply a set of equilibrium asset prices $p_{L}$ and $p_{K}$ relative to the numeraire; the purpose of this section is to derive equations for these equilibrium prices.

The individual's initial endowment is $W_{o i}=\bar{B}_{i}+p_{L} \bar{L}_{i}+p_{K} \bar{K}_{i}$ and must be redivided among new holdings $\left(B_{i}, L_{i}\right.$, and $\left.K_{i}\right)$ according to the wealth constraint:

$$
B_{i}+p_{L} L_{i}+p_{K} K_{i}=\bar{B}_{i}+p_{L} \bar{L}_{i}+p_{K} \bar{K}_{i}
$$

At the end of the "week," each unit of land is worth $P_{L}+R_{L}$, each unit of capital is worth $P_{K}+R_{K}$ and each unit of bills is worth $1+R_{B}$. Thus $R_{L} / P_{L}$ is the return per week per unit of land, $R_{K} / P_{K}$ is the rate of return on capital, and $R_{B}$ is the rate of interest. All of these are to be regarded as real after-tax rates of return. The returns to land and capital are uncer-

4. The idea of an expected time pattern of future inflation rates might be more realistic but would be more complex to analyze without adding any fundamentally new insights.

5. The effective stock of land can change through the loss of topsoil, forestations, etc

6. This is Tobin's $q$ value, the index of common stock prices per unit of real capital.

7. Poterba (1980) and Summers $(1980 c)$ have extended the type of analysis presented in Feldstein (1980; chaps. 10-12 above) to include an explicit capital stock adjustment process with feedback onto the path of asset prices. They assume certainty (or constant risk differentials) and therefore that the yields of all assets are always equated, at least up to a constant. 
tain, while the bill return is riskless. ${ }^{8}$ The individual's wealth at the end of the week is thus:

$$
W_{i}=\left(P_{L}+R_{L}\right) L_{i}+\left(P_{K}+R_{K}\right) K_{i}+\left(1+R_{B}\right) B_{i}
$$

If each individual has the same quadratic utility function, expected utility can be written as a linear combination of the mean and variance of $W_{i}$ :

$$
E\left[u\left(W_{i}\right)\right]=E\left(W_{i}\right)-0.5 \gamma \cdot \operatorname{var}\left(W_{i}\right)
$$

where $\gamma>0$ is a measure of risk aversion and the 0.5 is introduced to simplify subsequent calculations.

Equation (2) implies that

$$
E\left(W_{i}\right)=\bar{R}_{L} L_{i}+\bar{R}_{K} K_{i}+R_{B} B_{i}+W_{o i}
$$

where the bars over the $\bar{R}_{L}$ and $\bar{R}_{K}$ denote expected yields for the one week holding period. By using equation (1), this may be rewritten

$$
\begin{aligned}
E\left(W_{i}\right)= & \bar{R}_{L} L_{i}+\bar{R}_{K} K_{i}+R_{B}\left[p_{L}\left(\bar{L}_{i}-L_{i}\right)\right. \\
& \left.+p_{K}\left(\bar{K}_{i}-K_{i}\right)+\bar{B}_{i}\right]+W_{o i}
\end{aligned}
$$

Equation (2) also implies that

$$
\operatorname{var}\left(W_{i}\right)=\sigma_{L L} L_{i}^{2}+\sigma_{K K} K_{i}^{2}+\sigma_{L K} L_{i} K_{i}
$$

where $\sigma_{L L}$ and $\sigma_{K K}$ as the variances of the one week holding period returns and $\sigma_{K L}$ is the covariance.

The household's optimum portfolio is found by maximizing the value of expected utility in equation (3) subject to the constraint of equation (1). Using equations (5) and (6), this implies the first-order conditions:

$$
0=\bar{R}_{L}-R_{B} p_{L}-\gamma\left[\sigma_{L L} L_{i}+\sigma_{L K} K_{i}\right]
$$

and

$$
0=\bar{R}_{K}-R_{B} p_{K}-\gamma\left[\sigma_{K K} K_{i}+\sigma_{K L} L_{i}\right]
$$

The pair of asset demand equations may therefore be written:

$$
\gamma\left[\begin{array}{ll}
\sigma_{L L} & \sigma_{L K} \\
\sigma_{K L} & \sigma_{K K}
\end{array}\right]\left[\begin{array}{c}
L_{i} \\
K_{i}
\end{array}\right]=\left[\begin{array}{cc}
\bar{R}_{L} & -R_{B} p_{L} \\
\bar{R}_{K} & -R_{B} p_{K}
\end{array}\right]
$$

or

$$
\left[\begin{array}{l}
L_{i} \\
K_{i}
\end{array}\right]=\gamma^{-1}\left[\begin{array}{cc}
\sigma_{L L} & \sigma_{L K} \\
\sigma_{K L} & \sigma_{K K}
\end{array}\right]^{-1}\left[\begin{array}{l}
\bar{R}_{L}-R_{B} p_{L} \\
\bar{R}_{K}-R_{B} p_{K}
\end{array}\right]
$$

8. This reflects the assumption that the inflation rate for the current week is known even though the future inflation is uncertain. 
Since all of the investors are identical, each demands the same $L_{i}$ and $K_{i}$. Summing $L_{i}$ and $K_{i}$ over all individuals gives the total demand which must equal the total asset supplies: $N \bar{L}$ and $N \bar{K} \cdot{ }^{10}$ Thus

$$
\begin{aligned}
& {\left[\begin{array}{c}
\Sigma_{i} L_{i} \\
\Sigma_{i} K_{i}
\end{array}\right]=N \gamma^{-1}\left[\begin{array}{ll}
\sigma_{L L} & \sigma_{L K} \\
\sigma_{K L} & \sigma_{K K}
\end{array}\right]^{-1}} \\
& {\left[\begin{array}{l}
\bar{R}_{L}-R_{B} p_{L} \\
\bar{R}_{K}-R_{B} p_{K}
\end{array}\right]=\left[\begin{array}{l}
N \bar{L} \\
N \bar{K}
\end{array}\right]}
\end{aligned}
$$

Equation (10) can thus be solved explicitly for the equilibrium asset prices as functions of the expected yields, the covariance matrix, and the initial asset quantities:

$$
\left[\begin{array}{l}
p_{L} \\
p_{K}
\end{array}\right]=R_{B}^{-1}\left[\left(\begin{array}{l}
\bar{R}_{L} \\
\bar{R}_{K}
\end{array}\right)-\gamma\left[\begin{array}{ll}
\sigma_{L L} & \sigma_{L K} \\
\sigma_{K L} & \sigma_{K K}
\end{array}\right]\left(\begin{array}{c}
\bar{L} \\
\bar{K}
\end{array}\right)\right]
$$

or

$$
p_{L}=R_{B}^{-1}\left[\bar{R}_{L}-\gamma\left(\sigma_{L L} \bar{L}+\sigma_{L K} \bar{K}\right)\right]
$$

and

$$
p_{K}=R_{B}^{-1}\left[\bar{R}_{K}-\gamma\left(\sigma_{K L} \bar{L}+\sigma_{K K} \bar{K}\right)\right]
$$

\subsection{The Means and Variances of Asset Yields}

I turn now to the derivation of the mean real net-of-tax returns on the three assets and the corresponding covariance matrix.

Consider first the real net rate of return on bills. If the nominal short-term rate is $r$, the personal tax rate is $\theta$, and the actual current inflation rate is $\pi$, the real net-of-tax rate of return is

$$
R_{B}=(1-\theta) r-\pi
$$

Because the tax is levied on the nominal return, the real net-of-tax returns will vary with the rate of inflation. Ever since Irving Fisher's 1930 study, empirical studies have confirmed that the nominal interest rate changes approximately point for point with sustained changes in the rate of inflation;" in the current notation, $d r / d \pi=1$ is a reasonable approximation. This implies that $d R_{B} / d \pi=-\theta<0$; an increase in the inflation rate reduces the real net return on bills. For an inflation rate high enough, the real return can be negative. This is a particularly important feature of

9. I assume the conditions on the covariance matrix and the yield vector are such that $0 \leq$ $L_{i}$ and $0 \leq K_{i}$ and $P_{L} L_{i}+p_{K} K_{i} \leq p_{L} \bar{L}_{i}+p_{K} \bar{K}_{i}+\bar{B}_{i}$. These conditions must surely be fulfilled in an economy of identical individuals.

10. Since all individuals demand the same assets, $\bar{L}_{j}=\bar{L}_{j}$ for all $i, j$ and the subscript can be ignored.

11. See, e.g., Yohe and Karnovsky (1969) and Feldstein and Summers (1977). 
our tax system because it suggests that the usual assumption of equal yields on all assets may be wrong and a poor approximation when there is substantial inflation.

The return on a unit of land consists of an income return and a capital gain or loss. If the marginal physical product per unit of land (per week) is $F_{L}$, the net-of-tax marginal revenue product is $(1-\theta) p F_{L}$. Increases in the price of land are taxable capital gains. The capital gains tax rate is less than the tax rate on ordinary income and the effective tax rate is further reduced because capital gains are taxed only when the property is sold; I shall use the letter $c$ to denote the accrual-equivalent effective tax rate, i.e., the rate which, levied on accruals, would collect the same present value of taxes as the actual rate levied on realizations. If the increase in the price of land during the week is $\dot{p}_{L}$, the after-tax capital gain is $(1-c) \dot{p}_{L}$.

The total nominal return per unit of land is thus $(1-\theta) p F_{L}+(1-$ c) $\dot{p}_{L}$. Since a unit of land $\operatorname{costs} p_{L}$, the nominal return per dollar invested in land is $(1-\theta) p F_{L} / p_{L}+(1-c) \dot{p}_{L} / p_{L}$. The real rate of return is the difference between this nominal rate of return and the rate of inflation: $(1-\theta) p F_{L} / p_{L}+(1-c) \dot{p}_{L} / p_{L}-\pi$. Finally, the real return per unit of land $\left(R_{L}\right)$ is just the product of the real rate of return and the price per unit of land:

$$
R_{L}=(1-\theta) p F_{L}+(1-c) \dot{p}_{L}-\pi p_{L}
$$

There are two types of uncertainty about this return, corresponding to the income and capital gain components of the price change. Since the current price level is known, the income uncertainty is caused by the uncertain marginal physical product of land. If $\phi_{L}$ is the mean marginal physical product of land and $\widetilde{v}$ is the random component with the zero mean and variance $\sigma_{v v}$,

$$
F_{L}=\phi_{L}+\tilde{v}
$$

In a stationary equilibrium the price of land will rise at the same rate as the general price level: $\dot{p}_{L} / p_{L}=\pi$. Changes in the expected future rate of inflation or in the expected future value of any other factor that influences the value of land will cause the price of land to change by more or less than the current rate of inflation. The uncertain change in the price of land can be written without restriction as:

$$
\frac{\dot{p}_{L}}{p_{L}}=\pi+\widetilde{\epsilon}
$$

where $\widetilde{\epsilon}$ is a random variable with zero mean, variance $\sigma_{\epsilon \epsilon}$ and covariance $\sigma_{\nu \epsilon}$ with the random disturbance to productivity.

Substituting (15) and (16) into (14) yields: ${ }^{12}$

12. This is the natural extension to an economy with uncertainty of the return on land derived in equation (1.5) of Feldstein (1908a). 


$$
\begin{aligned}
R_{L} & =(1-\theta) p\left(\phi_{L}+\widetilde{v}\right)+(1-c)(\pi+\widetilde{\epsilon}) p_{L}-\pi p_{L} \\
& =(1-\theta) p \phi_{L}+(1-\theta) p \widetilde{v}-c \pi p_{L}+(1-c) p_{L} \widetilde{\boldsymbol{\epsilon}}
\end{aligned}
$$

The mean return per unit of land is thus

$$
\bar{R}_{L}=(1-\theta) p \phi_{L}-c \pi p_{L}
$$

The variance of this return is

$$
\begin{aligned}
\sigma_{L L}= & (1-\theta)^{2} p^{2} \sigma_{v v}+(1-c)^{2} p_{L}^{2} \sigma_{\epsilon \epsilon} \\
& +2(1-\theta)(1-c) p p_{L} \sigma_{\epsilon v}
\end{aligned}
$$

The return on reproducible capital also consists of an income return and a change in the price of the asset. Because the tax rules are based on nominal accounting definitions, a rise in the rate of inflation increases the effective tax rate on the real income from reproducible capital. ${ }^{13}$ This is due primarily to the required use of historic cost depreciation but also reflects the method of inventory accounting. ${ }^{14}$ If the marginal physical product per unit of capital is $F_{K}$, the net-of-tax marginal revenue product in the absence of inflation can be written $(1-\theta) p F_{K}{ }^{15}$ It is convenient to approximate the extra tax burden per unit of capital as proportional to the rate of inflation; the real return per unit of capital is thus depressed by $\lambda \pi p$ at current prices. The real net of tax income per unit of capital is thus $(1-\theta) p F_{K}-\lambda \pi p$. If the increase in the market price of capital ${ }^{16}$ during the week is $\dot{p}_{K}$, the net-of-tax capital gain $(1-c) \dot{p}_{K}$. The total nominal return per unit of capital is thus $(1-\theta) p F_{K}-\lambda \pi p+(1-c) \dot{p}_{K}$ and the corresponding real return per unit of capital is:

$$
R_{K}=(1-\theta) p F_{K}-\lambda \pi p+(1-c) \dot{p}_{K}-\pi p_{K}
$$

The income uncertainty of the return on capital reflects the uncertain marginal product of capital and can be represented by:

$$
F_{K}=\phi_{K}+\widetilde{v}
$$

where $\widetilde{v}$ has mean zero and variance $\sigma_{v v}$. The uncertain change in the price of existing capital assets can be written

$$
\frac{\dot{p}_{K}}{p_{K}}=\pi+\widetilde{\omega}
$$

13. Recall that this analysis uses "reproducible capital" to refer to business capital and tgnores owner-occupied real estate.

14. See Feldstein and Summers (1979; chap. 8 above) and Feldstein (1980b; chap. 10 above) for a discussion of how higher tnflation increases the effective tax rate on the income of nonfinancial corporations and of their equity owners.

15. This ignores the separate corporate income tax and the differential treatment of dividends and retained earnings. Recognizing these would complicate the analysis without changing anything fundamental

16. This perhaps is best thought of as the market price of common stock, i.e., claims to the existing capital stock rather than new capital goods. 
where $\widetilde{\omega}$ has variance $\sigma_{\omega \omega \omega}$ and covariance with $\widetilde{v}$ of $\sigma_{v \omega}$.

Substituting (21) and (22) into (20) yields:

$$
\begin{aligned}
R_{K} & =(1-\theta) p\left(\phi_{K}+\widetilde{v}\right)-\lambda \pi p+(1-c)(\pi+\widetilde{\omega}) p_{K}-\pi p_{K} \\
& =(1-\theta) p \phi_{K}+(1-\theta) p \widetilde{v}-\lambda \pi p-c \pi p_{K}+(1-c) p_{K} \widetilde{\omega}
\end{aligned}
$$

The mean return per unit of capital is thus:

$$
\bar{R}_{K}=(1-\theta) p \phi_{K}-\lambda \pi p-c \pi p_{K}
$$

and the variance is

$$
\begin{aligned}
\sigma_{K K}= & (1-\theta)^{2} p^{2} \sigma_{v v}+(1-c)^{2} p_{K} \sigma_{\omega \omega} \\
& +2(1-\theta)(1-c) p p_{K} \sigma_{v \omega}
\end{aligned}
$$

The covariance between the returns on capital and land depends in general on the full covariance matrix of all four random effects:

$$
\begin{aligned}
\sigma_{K L}=E\{[(1-\theta) p \widetilde{v} & \left.\left.+(1-c) p_{L} \epsilon\right]\left[(1-\theta) p \widetilde{v}+(1-c) p_{K} \widetilde{\omega}\right]\right\} \\
=(1-\theta)^{2} p^{2} \sigma_{\nu v} & +(1-\theta)(1-c) p p_{K} \sigma_{\nu \omega} \\
& +(1-c)(1-\theta) p p_{L} \sigma_{\epsilon v} \\
& +(1-c)^{2} p_{L} p_{K} \sigma_{\omega \epsilon}
\end{aligned}
$$

\subsection{The Price Equations}

The means and covariance matrix of the returns on land and capital can be used with equation (12a-b) to obtain explicit price equations for land and capital. It is useful to begin by substituting the mean values $R_{B}$ and $\bar{R}_{L}$ into (12) to obtain the price of land:

$$
p_{L}=\frac{(1-\theta) p \phi_{L}-c \pi p_{L}-\gamma\left(\sigma_{L L} \bar{L}+\sigma_{L K} \bar{K}\right)}{(1-\theta) r-\pi}
$$

Collecting and rearranging terms yields:

$$
\frac{p_{L}}{p}=\frac{(1-\theta) \phi_{L}}{(1-\theta) r-(1-c) \pi+\gamma p_{L}^{-1}\left(\sigma_{L L} \bar{L}+\sigma_{L K} \bar{K}\right)}
$$

There are several significant things to notice about this expression for the real price of land $\left(p_{L} / p\right)$. In the absence of risk aversion $(\gamma=0)$ and inflation $(\pi=0)$, the real price of land is just the discounted value of the expected return per unit of land, i.e., $p_{L} / p=\phi_{L} / r$. If there is inflation but no risk aversion, the relationship is more complex; the perpetuity at $\phi_{L}$ is discounted by $r-[(1-c) /(1-\theta)] \pi$. Since $(1-c) /(1-\theta)<1$, this "net discount rate" can easily become "negative." That is, as $\pi$ rises, $r-[(1$ $-c) /(1-\theta) \pi]$ approaches zero and the implied relative price of land becomes indefinitely large. When $(1-\theta) r<(1-c) \pi$, the value of $p_{L} / p$ 
"passes through" infinity and becomes apparently negative. More generally, for many plausible tax parameters, the relative price of land is implausibly sensitive to changes in $\pi$.

These results show the importance of explicitly recognizing the role of uncertainty and risk aversion in determining $p_{L} / p$. Equation (28) shows that risk aversion can eliminate the anomalous results. With $\gamma\left(\sigma_{L L} \bar{L}+\sigma_{L K} \bar{K}\right)>0$ in the denominator, relative asset prices are not nearly so sensitive to differences in the mean real net rates of return.

A more complete characterization of the real price of land is obtained if $\sigma_{L L}$ and $\sigma_{L K}$ are rewritten in terms of the underlying variances and covariances. The essential features of the analysis are preserved but the analysis is simplified by assuming that the income disturbances ( $\widetilde{v}$ and $\widetilde{v}$ ) are independent of each other and of the price disturbances ( $\widetilde{\epsilon}$ and $\widetilde{\omega})$. Such an assumption would be reasonable if investors knew that the disturbances $\widetilde{v}$ and $\widetilde{v}$ are serially independent so that a disturbance in one period has no implications about future values of $F_{L}$ and $F_{K}$. With this simplifying assumption, the relevant variances and covariances of section 13.2 become:

$$
\begin{aligned}
\sigma_{L L} & =(1-\theta)^{2} p^{2} \sigma_{\nu v}+(1-c)^{2} p_{L}^{2} \sigma_{\epsilon \epsilon} \\
\sigma_{K K} & =(1-\theta)^{2} p^{2} \sigma_{v v}+(1-c)^{2} p_{K}^{2} \sigma_{\omega \omega}
\end{aligned}
$$

and

$$
\sigma_{L K}=(1-c)^{2} p_{L} p_{K} \sigma_{\omega \epsilon}
$$

Substituting these values into (28) yields

$$
\begin{aligned}
\frac{p_{L}}{p}= & \frac{(1-\theta) \phi_{L}}{(1-\theta) r-(1-c) \pi+\gamma p_{L}^{-1}\left\{\left[(1-\theta)^{2} p^{2} \sigma_{\nu v}\right.\right.} \\
& \frac{(1-\theta) \phi_{L}}{\left.\left.+(1-c)^{2} p_{L}^{2} \sigma_{\epsilon \epsilon}\right] \bar{L}+(1-c)^{2} p_{L} p_{K} \sigma_{\omega \epsilon} \bar{K}\right\}}
\end{aligned}
$$

or

$$
\begin{aligned}
\frac{p_{L}}{p}= & \frac{(1-\theta) \phi_{L}}{(1-\theta) r-(1-c) \pi+\gamma\left\{\left[(1-\theta)^{2}\left(p / p_{L}\right)^{2} \sigma_{\nu v}\right.\right.} \\
& \frac{(1-\theta) \phi_{L}}{\left.\left.+(1-c)^{2} \sigma_{\epsilon \epsilon}+(1-c)^{2} \sigma_{\epsilon \epsilon}\right] p_{L} \bar{L}+(1-c)^{2} \sigma_{\omega \epsilon} p_{K} \bar{K}\right\}}
\end{aligned}
$$

In this form, the real price of land is defined as a quadratic function of tax rates, rates of return, the expected inflation rate, and the total wealth in land and capital. If the income risk is ignored $\left(\sigma_{v v}=0\right)$, the real price of land assumes the simple form: 


$$
\frac{p_{L}}{p}=\frac{(1-\theta) \phi_{L}}{(1-\theta) r-(1-c) \pi+\gamma(1-c)^{2}\left(\sigma_{\epsilon \epsilon} p_{L} \bar{L}+\sigma_{\epsilon \omega} p_{K} \bar{K}\right)}
$$

This case is also substantively interesting because the price risk can generally be expected to be large relative to the income risk and because uncertainty about the future inflation rate contributes to the price risk but not the income risk.

The analogous equation for the real market price of capital is

$$
\frac{p_{K}}{p}=\frac{(1-\theta) \phi_{K}-\lambda \pi}{(1-\theta) r-(1-c) \pi+\gamma(1-c)^{2}\left(\sigma_{\omega \omega} p_{K} \bar{K}+\sigma_{\epsilon \omega} p_{L} \bar{L}\right)}
$$

\subsection{Some Comparative Static Analyses}

Equations (34) and (35) can be used to examine how the real prices of land and capital respond to changes in inflation, the uncertainty of future inflation, and the like. Since the stock of capital is assumed to remain constant,${ }^{17}$ the results can, of course, only indicate the direction and not the magnitude of the change.

The derivative of $p_{L} / p$ with respect to the expected inflation rate is easily shown to be:

$$
\begin{aligned}
\frac{d\left(p_{L} / p\right)}{d \pi}= & -\frac{\left(p_{L} / p\right)^{2}}{(1-\theta) \phi_{L}}\left\{(1-\theta) \frac{d r}{d \pi}-(1-c)\right. \\
& +\gamma(1-c)^{2} \sigma_{\epsilon \epsilon} \frac{d V_{L}}{d \pi} \\
& \left.+\gamma(1-c)^{2} \sigma_{\epsilon \omega} \frac{d V_{K}}{d \pi}\right\}
\end{aligned}
$$

where $V_{L}=p_{L} \bar{L}$ and $V_{K}=p_{K} \bar{K}$. Note first that, in the absence of risk aversion, the effect of inflation on the real price of land is positive if $(d r / d \pi)<(1-c) /(1-\theta)$. Since $c<\theta$, this will clearly be satisfied whenever $d r / d \pi \leq 1$. During the increasing inflation of the $1960 \mathrm{~s}$ and 1970 s, the nominal interest rate rose by approximately the rise in the rate of inflation, causing the real net interest rate to fall by $-\theta d \pi$. In contrast, the real return on land falls only because of the smaller rate of capital gains tax on the nominal appreciation in the value of the land. Since the extra tax on bills per dollar of capital would exceed the extra tax on land, the price of land rises in the absence of uncertainty in order to equalize the yields.

Introducing uncertainty leaves this conclusion unchanged but suggests that the magnitude of the effect may be reduced. If $d\left(p_{L} / p\right) / d \pi>0$,

17. See above, p. 231. 
$d V_{L} / d \pi>0$ since $V_{L}=p_{L} \bar{L}$ and $\overline{\mathrm{L}}$ is constant. This positive term offsets some of the magnitude of the pure tax and interest rate effect. The economic reason for this is that as $p_{L}$ rises the investor has relatively more wealth in this form which in turn raises the risk premium that the investor requires to hold even more land or, equivalently, which reduces the demand for more land and therefore the real price of land.

If the primary reason for the covariance between the unanticipated changes in the prices of land and capital $\left(\sigma_{\epsilon \omega}\right)$ are the unanticipated changes in inflation, the term $\sigma_{\epsilon \omega} d V_{K} / d \pi$ is also likely to be positive, further reducing $d\left(p_{L} / p\right) / d \pi$ but nevertheless leaving it positive. For example, $d p_{L} / d \pi>0$ and $d p_{K} / d \pi<0$ imply $\sigma_{\epsilon \omega}<0$ and $d V_{K} / d \pi<0$ and therefore that $\sigma_{\epsilon \omega} d V_{K} / d \pi>0$. Similarly, $d p_{L} / d \pi>0$ and $d p_{K} / d \pi>0$ imply $\sigma_{\omega \omega}>0$ and $d V_{K} / d \pi>0$ and therefore again $\sigma_{\epsilon \omega} d V_{K} / d \pi>0$. The economic reason (in the relevant case in which $d p_{K} / d \pi<0$ ) is that inflation reduces the value of the investors' reproducible capital and, since the return on capital is negatively correlated with the return on land, reduces the demand for land and therefore its price.

The effect of uncertainty is nevertheless to dampen the effect of inflation and not to reverse it. To see this, note that the opposite implies a contradiction. If $d p_{L} / d \pi<0, \sigma_{\epsilon \epsilon}\left(d V_{L} / d \pi\right)<0$ which implies an even larger positive value of $d p_{L} / d \pi$.

A similar analysis shows that a higher rate of inflation reduces the real value of capital ${ }^{18}$ and that the uncertainty and risk aversion again dampen the magnitude of the effect.

Consider now the effect of an increase in the uncertainty of the future inflation rate. This increases $\sigma_{\epsilon \epsilon}, \sigma_{\omega \omega}$, and $\left|\sigma_{\epsilon \omega}\right|$. The relative increase in each term depends on the extent to which uncertainty and inflation is the source of the uncertainty about asset prices. Two extremes will illustrate the possible results. If most of the variation in the real price of land reflects variation in anticipated inflation while little of the variation in the inflation uncertainty will raise $\sigma_{\epsilon \epsilon}$ while leaving $\sigma_{\omega \omega}$ essentially unchanged. Moreover, if inflation is not a major source of $\sigma_{\omega \omega}$, it is possible (although not necessary) that $\sigma_{\epsilon \omega}=0$. Total differentiation of equation (34) with respect to $p_{L}$ and $\sigma_{\epsilon \epsilon}$ with $\sigma_{\epsilon \omega}=0$ implies that $d p_{L} / d \sigma_{\epsilon \epsilon}<0$, i.e., an increase in inflation uncertainty unambiguously reduces $p_{L}$ while leaving $p_{K}$ unchanged.

In contrast, consider the case in which inflation uncertainty is equally important for $\sigma_{\epsilon \epsilon}$ and $\sigma_{\omega \omega \omega}$ and $\sigma_{\epsilon \omega}<0$. If an increase in inflation uncertainty raises $\sigma_{\epsilon \epsilon}$ and $\sigma_{\omega \omega}$ by equal amounts and leaves the correlation between $\epsilon$ and $\omega$ unchanged, (34) and (35) imply that an increase in inflation uncertainty reduces both $p_{L}$ and $p_{K}$. Investors respond to the

18. This depends on the relative magnitudes of the historic cost depreciation effect and the real interest rate effect. For an analysis with realistic parameters, see Feldstein (1980b; chap. 10 above). 
increased uncertainty by demanding less land and capital and more of the riskless nominal asset.

More generally, the response of relative asset prices to an increase in inflation uncertainty will depend on the relative extent to which $\sigma_{\epsilon \epsilon}, \sigma_{\omega \omega}$, and $\sigma_{\epsilon \omega}$ are changed. An increase in inflation uncertainty might cause the real price of land to rise if investors wish to substitute both land and bills for capital.

\subsection{Conclusion}

This paper has focused on the specific question of how changes in expected inflation and in its uncertainty affect the real prices of land and of reproducible capital. The analysis shows how an explicit portfolio choice framework can be applied to derive asset price equations and how, in this framework, the interaction of taxes and increased inflation causes a rise in the real value of land and a fall in the real value of corporate equities.

Two more general points are worth noting. First, the analysis shows the inappropriateness of the common assumption that inflation is neutral, i.e., that it does not alter real magnitudes. When there are taxes on capital income, this is false and inflation can have substantial real effects.

Second, the traditional assumption that prices adjust until net-of-tax yields are equal may be very misleading. In the examples shown here, the existence of a finite price for land depends on the uncertainty of the asset yields.

This paper has shown that an explicit utility maximization model of portfolio choice can be applied to analyzing the effects of changes in the rate of inflation. A natural next step is to embed this analysis in a more general dynamic framework in which changes in the price of capital change the supply of new capital goods and therefore the future path of the real marginal products of capital and land. 
IV

\author{
The Effect \\ on Investment
}


